\title{
Cochrane
(áxico
}

\section{Inhibidores de la aromatasa para estatura baja en niños y adolescentes de sexo masculino}

\author{
Leticia A. Barajas-Nava ${ }^{1 *}$ y M. Fernanda Castilla-Peón ${ }^{2}$ \\ ${ }^{1}$ Unidad de Investigación de Medicina Basada en Evidencias, Centro Asociado Cochrane; ${ }^{2}$ Ediciones Médicas, Hospital Infantil de México Federico \\ Gómez. Ciudad de México, México
}

\section{Resumen}

Los inhibidores de la aromatasa son fármacos disponibles por vía oral que inhiben la conversión de la testosterona en estradiol. Los estrógenos desempeñan un papel esencial en la maduración del cartílago de crecimiento y del cierre epifisario, evento que marca el final del proceso de crecimiento esquelético. Por este motivo, los inhibidores de la aromatasa se han probado como una intervención para mejorar la talla en niños y adolescentes de sexo masculino mediante el retraso en la maduración esquelética al disminuir la concentración de estradiol en la placa de crecimiento. Se resumen los resultados de una revisión sistemática Cochrane en la cual se evaluaron la eficacia y la seguridad de los inhibidores de la aromatasa para el tratamiento de la estatura baja en niños.

Palabras clave: Inhibidores de la aromatasa. Niños varones. Talla adulta. Revisión sistemática. Metaanálisis.

\section{Aromatase inhibitors for short stature in male children and adolescents}

\section{Abstract}

Aromatase inhibitors are orally administered drugs that inhibit the conversion of testosterone to estradiol. Estrogens have an important role in growth plate maturation and epiphyseal closure. Thus, aromatase inhibitors have been used to improve final height in male children and adolescents by delaying skeletal maturation through a decrease in estradiol concentration. The results of a Cochrane systematic review evaluating the efficacy and safety of aromatase inhibitors for the treatment of short stature in children are summarized below.

Keywords: Aromatase inhibitors. Male children. Adult height. Systematic review. Meta-analysis.

Available online: 27-07-2021 Bol Med Hosp Infant Mex. 2021;78(5):457-460

www.bmhim.com 1665-1146/@ 2020 Hospital Infantil de México Federico Gómez. Published by Permanyer. This is an open access article under the CC BY-NC-ND license (http://creativecommons.org/licenses/by-nc-nd/4.0/). 


\section{Introducción}

Una revisión Cochrane evaluó los efectos de los inhibidores de la aromatasa en varones menores de 16 años con talla baja idiopática, deficiencia de hormona del crecimiento o retraso constitucional del crecimiento y del desarrollo'.

Las causas más frecuentes de talla baja son el retraso constitucional del crecimiento y el retraso del desarrollo puberal, así como la talla baja familiar. Estas condiciones no están determinadas por ninguna enfermedad o deficiencia nutricional.

Con el fin de disminuir el daño psicológico que pudiera causar el tener talla baja, se han implementado diversos tratamientos para el aumento de la talla adulta. Las terapias más utilizadas son la hormona del crecimiento, que ha demostrado una eficacia modesta ${ }^{2,3}$, y los esteroides sexuales, que no parecen mejorar la talla adulta prevista. Por otro lado, los análogos de la hormona liberadora de gonadotropina, que son el estándar de atención para el tratamiento de la pubertad precoz central, no han demostrado su eficacia en el aumento de la estatura adulta cuando se utilizan fuera del contexto de la pubertad precoz, y además son un tratamiento costoso e invasivo.

En las últimas décadas se han utilizado los inhibidores de la aromatasa para algunas afecciones en niños y adolescentes (pubertad precoz periférica, hiperplasia suprarrenal congénita, ginecomastia puberal, estatura baja). Así mismo, las intervenciones para mejorar los resultados en la talla final de los varones incluyen tanto los inhibidores de la aromatasa de tercera generación (anastrozol y letrozol) como los moduladores selectivos de los receptores de estrógenos (tamoxifeno), con los cuales se ha observado que la duración del tratamiento influye en la talla final.

Los inhibidores de la aromatasa previenen la conversión de la testosterona a estrógenos, los cuales provocan el cierre de las placas de crecimiento en los huesos largos al final de la pubertad. En los varones, el uso de este fármaco puede prolongar el periodo de crecimiento y con ello la estatura en la adultez. En las mujeres, los estrógenos son esenciales, por lo que no es apropiado el uso de estos fármacos. La vía de administración oral y la capacidad de permitir que la pubertad continúe en un momento fisiológicamente apropiado hacen que los inhibidores de la aromatasa sean una intervención atractiva para retrasar la maduración esquelética y aumentar el potencial de altura. Actualmente no están aprobados para tratar la talla baja, pero se utilizan fuera de la indicación permitida.
Por lo anterior, esta revisión Cochrane evaluó los efectos de los inhibidores de la aromatasa en niños y adolescentes varones de estatura baja.

\section{Métodos}

La revisión Cochrane incluyó ensayos clínicos controlados aleatorizados y quasi-aleatorizados que evaluaron un inhibidor de la aromatasa en comparación con placebo o con no tratamiento en varones de 16 años o menos con talla baja idiopática, deficiencia de hormona de crecimiento o retraso constitucional del crecimiento y del desarrollo, que hubiesen recibido al menos 12 meses de tratamiento'.

Los resultados principales evaluados en la revisión fueron la estatura final o casi final, la calidad de vida relacionada con la salud, los eventos adversos, los valores de testosterona y la densidad y la morfología óseas, entre otros.

La revisión se realizó de acuerdo con la metodología recomendada en el Manual Cochrane para revisiones sistemáticas de intervenciones 4 .

Se realizaron búsquedas en The Cochrane Library, Cochrane Central Register of Controlled Trials (CENTRAL), MEDLINE, EMBASE, International Clinical Trials Registry Platform (ICTRP) de la World Health Organization (WHO) (apps.who.int/trialsearch/), Australian New Zealand Clinical Trials Registry, clinicaltrials.gov y EU Clinical Trials Register (EU-CTR) hasta agosto de 2014, sin restricción de idioma, utilizando filtros metodológicos. Adicionalmente se revisaron las listas de referencias de los artículos recuperados e incluidos, las bases de datos de empresas farmacéuticas y otras fuentes de literatura gris.

Dos revisores examinaron de forma independiente los criterios de inclusión para todos los artículos identificados y recuperados, así como la extracción y la gestión de datos y la evaluación del riesgo de sesgo de cada ensayo incluido. Los desacuerdos se resolvieron por consenso o mediante consultar a un tercer revisor. Para el análisis de los datos se utilizaron la razón de momios o los cocientes de riesgos para datos dicotómicos y la diferencia de medias para datos continuos, con intervalos de confianza del 95\%. Se evaluó la heterogeneidad (inconsistencia) mediante el uso de un valor estándar de $\chi^{2}$. Para la elaboración de los análisis se utilizó el software estadístico de la Colaboración Cochrane: Review Manager (RevMan) Versión 5.35. 


\section{Resultados}

En total, la revisión Cochrane incluyó cuatro ensayos clínicos controlados aleatorizados (13 publicaciones), los cuales analizaron 207 niños y adolescentes varones con estatura baja, retraso constitucional del crecimiento y de la pubertad, o ambos, o bien con deficiencia de hormona del crecimiento. A estos pacientes se les administró letrozol $(2.5 \mathrm{mg})$ en tres estudios (58 participantes) 0 anastrozol (1 mg) en un estudio (26 participantes) durante 12 a 36 meses, en comparación con placebo'. Los estudios se realizaron en la consulta ambulatoria de endocrinología pediátrica. Los resultados reportados en la revisión fueron los siguientes: talla final o casi final, velocidad de crecimiento, etapa puberal, edad ósea, densidad mineral ósea, hormonas sexuales y eventos adversos.

El aumento de estatura a corto o largo plazo solo fue reportado en dos estudios. Un estudio reportó que la talla final del grupo de letrozol/testosterona fue $7.5 \mathrm{~cm}$ más alta que la del grupo de placebo/testosterona (177.6 cm frente a $170.1 \mathrm{~cm}$, respectivamente). Sin embargo, en el grupo de letrozol/testosterona los participantes eran $7.2 \mathrm{~cm}$ más altos al inicio del estudio. El otro estudio reportó que la talla casi final en el grupo de letrozol/testosterona fue $6.9 \mathrm{~cm}$ más alta que la del grupo de placebo/testosterona $(175.8 \mathrm{~cm}$ frente a $169.1 \mathrm{~cm}$, respectivamente).

En un estudio, la velocidad de crecimiento fue más rápida en los primeros 5 meses de tratamiento en el grupo de placebo/testosterona que en el grupo de letrozol/testosterona. Al final del estudio, se encontró un incremento del $33 \%$ en los valores de testosterona en el grupo de placebo/testosterona y del $420 \%$ en el grupo de letrozol/testosterona. A pesar de que no se reportan efectos adversos graves, una publicación informó sobre el desarrollo de anomalías morfológicas de las vértebras en el $45 \%$ de los sujetos del grupo con letrozol comparado con placebo.

La calidad de los estudios fue muy variable, presentando en su mayoría un riesgo de sesgo poco claro. De igual forma, la evaluación mediante el sistema GRADE (Grading of Recommendations Assessment, Development, and Evaluation) mostró que la evidencia era de moderada a baja calidad.

\section{Conclusions}

En conclusión, los ensayos aleatorizados evaluados en la revisión indican que los inhibidores de la aromatasa mejoran los resultados de crecimiento a corto plazo. Sin embargo, no existe evidencia suficiente para asegurar un aumento de estatura hasta la edad adulta.

Hasta ahora se desconoce el momento óptimo para iniciar la terapia con inhibidores de la aromatasa. No obstante, el hallazgo de anomalías vertebrales en niños prepúberes sugiere que puede ser mejor tratarlos durante la pubertad. Así mismo, se desconocen la duración óptima del tratamiento y la seguridad de su uso a largo plazo.

Se requieren nuevos ensayos clínicos con una adecuada calidad metodológica y que consideren poblaciones homogéneas y con criterios diagnósticos bien establecidos.

\section{Implicaciones para la práctica}

Los inhibidores de la aromatasa son una opción en el manejo de la talla baja en sujetos con mal pronóstico de talla final y en los que se considere que la interrupción de la pubertad y de la acción androgénica podría agravar de manera importante el malestar psicosocial derivado de la talla baja.

Si bien la evidencia encontrada en esta revisión es muy débil para sustentar la efectividad de los inhibidores de la aromatasa en el incremento de la talla final, un ensayo clínico reciente no incluido en la revisión aporta resultados en el mismo sentido que los estudios presentados ${ }^{6}$.

Es importante evaluar los posibles y limitados beneficios de los inhibidores de la aromatasa en la talla final y en el bienestar psicosocial de cara a los potenciales efectos adversos óseos y metabólicos, así como la falta de información sobre los efectos a largo plazo en la función metabólica y reproductiva.

\section{Responsabilidades éticas}

Protección de personas y animales. Los autores declaran que para esta investigación no se han realizado experimentos en seres humanos ni en animales.

Confidencialidad de los datos. Los autores declaran que en este artículo no aparecen datos de pacientes.

Derecho a la privacidad y consentimiento informado. Los autores declaran que en este artículo no aparecen datos de pacientes.

\section{Conflicto de intereses}

Los autores declaran no tener ningún conflicto de intereses. 


\section{Financiamiento}

Ninguno.

\section{Bibliografía}

1. McGrath N, O'Grady MJ. Aromatase inhibitors for short stature in male children and adolescents. Cochrane Database Syst Rev. 2015;(8):CD010888

2. Paltoglou G, Dimitropoulos I, Kourlaba G, Charmandari E. The effect of treatment with recombinant human growth hormone (rhGH) on linear growth and adult height in children with idiopathic short stature (ISS): a systematic review and meta-analysis. J Pediatr Endocrinol Metab. 2020;33:1577-88.

3. Deodati A, Cianfarani S. Impact of growth hormone therapy on adult height of children with idiopathic short stature: systematic review. BMJ. 2011;342:c7157.

4. Higgins JPT, Green S, editores. Cochrane Handbook for Systematic Reviews of Interventions Version 5.1.0 [updated March 2011]. The Cochrane Collaboration; 2011. Disponible en: www.cochrane-handbook.org.

5. The Nordic Cochrane Centre, The Cochrane Collaboration. Review Manager (RevMan) Version 5.3. Copenhagen: The Nordic Cochrane Centre, The Cochrane Collaboration; 2014.

6. Mauras N, Ross JL, Gagliardi P, Yu YM, Hossain J, Permuy J, et al. Randomized trial of aromatase inhibitors, growth hormone, or combination in pubertal boys with idiopathic, short stature. J Clin Endocrinol Metab. 2016;101:4984-93. 